\title{
Corela
}

Cognition, représentation, langage

HS-11 | 2012

RJC Cotexte, contexte, situation

\section{Influence de la situation sur les interactions mère- enfant dysphasique et mère-enfant tout-venant}

Christine Da Silva, Stefano Rezzonico, Cristina Corlateanu, Séverine Gendre et Juliane Ingold

\section{OpenEdition}

\section{Journals}

\section{Édition électronique}

URL : http://journals.openedition.org/corela/2349

DOI : $10.4000 /$ corela. 2349

ISSN : $1638-573 \mathrm{X}$

\section{Éditeur}

Cercle linguistique du Centre et de l'Ouest - CerLICO

\section{Référence électronique}

Christine Da Silva, Stefano Rezzonico, Cristina Corlateanu, Séverine Gendre et Juliane Ingold, « Influence de la situation sur les interactions mère-enfant dysphasique et mère-enfant tout-venant », Corela [En ligne], HS-11 | 2012, mis en ligne le 02 avril 2012, consulté le 21 avril 2019. URL : http:// journals.openedition.org/corela/2349 ; DOI : 10.4000/corela.2349

Ce document a été généré automatiquement le 21 avril 2019

\section{(i) (2)}

Corela - cognition, représentation, langage est mis à disposition selon les termes de la licence Creative Commons Attribution - Pas d'Utilisation Commerciale - Partage dans les Mêmes Conditions 4.0 International. 


\title{
Influence de la situation sur les interactions mère-enfant dysphasique et mère-enfant tout-venant
}

\author{
Christine Da Silva, Stefano Rezzonico, Cristina Corlateanu, Séverine \\ Gendre et Juliane Ingold
}

\section{Introduction}

1 Cet article présente les premiers résultats d'une étude sur la manière dont les mères et les enfants interagissent ensemble selon diverses activités langagières. Ici, nous nous intéressons à des enfants présentant des troubles spécifiques du développement du langage âgés entre 5 et 7 ans.

2 Les troubles dysphasiques se caractérisent principalement par des troubles au niveau de la structure de la langue et ils sont très hétérogènes (Cf. les classifications de Rapin et Allen, 1983 et de Gérard, 1993). Plus spécifiquement, les enfants présentant une dysphasie phonologique - syntaxique (syndrome le plus fréquent) manifestent un déficit des capacités expressives dont une altération majeure de la phonologie et de la syntaxe (agrammatisme, dyssyntaxie).

3 Dans la littérature, on tend à distinguer les enfants présentant des troubles au niveau de la structure de la langue des enfants présentant des troubles pragmatiques (Bishop, 2000, Rapin et Allen, 1983). Cependant, on peut se demander si les troubles linguistiques peuvent affecter les capacités pragmatiques et notamment la participation des enfants dysphasiques au dialogue. Ainsi, certaines études se sont intéressées à la relation entre les troubles linguistiques et les troubles pragmatiques. (Cf. Hupet 1996, pour une synthèse) et tentent d'observer si les enfants dysphasiques présentent ou non des troubles pragmatiques (de Weck et Rosat, 2003).

4 Dans la littérature, les troubles pragmatiques mis en évidence chez les enfants dysphasiques concernent principalement l'implication dans l'échange verbal (Conti- 
Ramsden \& Gunn, 1986). Les enfants dysphasiques sont souvent décrits comme étant passifs (Leonard, 1986), ayant des difficultés à gérer une conversation (Conti-Ramsden, 1994), et /ou à donner des réponses appropriées à leur interlocuteur (Bishop et al., 2000). Par exemple, les enfants produisent davantage des interventions minimales principalement en réponse aux interventions de leurs interlocuteurs et ce de manière plus fréquente que leurs paires d'âge (Conti-Ramsden, Hutcheson et Grove, 1995). Par conséquent, les adultes ont alors tendance à prendre une place plus importante dans le discours, notamment en produisant plus d'initiatives afin de maintenir la conversation (Conti-Ramsden et Friel-Patti, 1984). Afin de vérifier ces aspects, diverses études ont été menées sur cette population, notamment sur la gestion des tours de parole, des thèmes (Radford \& Tarplee 2000), les pannes conversationnelles (Fujiki, Brinton \& Sonnenberg, 1990), les demandes de clarifications (Brinton, Fujiki et Sonnenberg, 1988), ou sur la pertinence des réactions des enfants dysphasiques (Brinton et Fujiki, 1982; Bishop et Adams, 1989).

5 Par ailleurs, certaines études se sont intéressées aux éventuels troubles discursifs des enfants dysphasiques. Elles ont montré que même si les enfants dysphasiques présentent une certaine sensibilité aux variations des conditions de production, ils ont des difficultés à passer de la polygestion à la monogestion lorsque la situation l'exige. De plus, la participation verbale des enfants dysphasiques au dialogue diffère selon le genre de discours (de Weck \& Rosat 2003). En effet, ils manifestent une plus grande habilité interactionnelle dans le cadre de discours ancrés dans la situation matérielle de production (en opposition avec des situations plus difficiles qui nécessitent l'évocation de référents absents et la création d'origine temporelle comme pour la narration et le récit d'expériences personnelles). Ceci montre que les enfants ont plus de difficultés pour entrer dans certains genres que dans d'autres (de Weck 1996).

6 Au vu de ces divers résultats, on peut donc se demander si la situation d'interaction peut influencer la manière dont les enfants dysphasiques participent à la co-construction du dialogue avec leurs mères et si cette participation diffère de celle des enfants sans troubles du langage. L'intérêt principal de cette étude est d'observer les caractéristiques variées de ces deux activités et l'effet éventuel fournir une base de réflexion aux cliniciens pour l'évaluation et l'analyse des conduites des enfants et des mères en interaction. Ces connaissances pourront également contribuer au développement de la guidance parentale.

\section{Méthodologie}

Nous présentons ci-dessous la population étudiée, les situations d'interaction avec leurs caractéristiques spécifiques, les conditions de recueil des données ainsi que les axes d'analyse empruntés afin de mettre en évidence les conduites dialogiques des dyades.

\subsection{Population}

8 Nous travaillons ici sur un sous-ensemble de la population de la recherche qui est composée de huit enfants âgés entre 5 et 7 ans : quatre enfants présentant des troubles spécifiques du développement du langage (dysphasie phonologique-syntaxique, TDL par la suite) et quatre enfants tout-venant (TV, par la suite). 
9 Les enfants TV ont été appariés aux enfants TDL selon l'âge chronologique (avec un mois d'écart au maximum), le sexe et l'origine (Suisse Romande ou France). De plus, afin d'objectiver les troubles du développement du langage chez les enfants dysphasiques et leur absence chez les enfants des groupes contrôles, tous ont passé des épreuves de production et de compréhension du langage issues des «Nouvelles épreuves pour l'Examen du Langage » (N-EEL) (Chevrie-Muller \& Plaza, 2001).

\subsection{Situations de communication}

10 Dans le domaine de l'acquisition du langage et de la pathologie, diverses recherches défendent l'hypothèse que les enfants actualisent leurs capacités langagières de manière différente selon les situations (de Weck, 1996 ; Dolz, Pasquier, \& Bronckart, 1993). Ainsi, nous nous sommes donc intéressés à deux situations de communication : une situation de lecture de livre et une situation de jeu symbolique.

11 Dans la situation de lecture de livre, la mère et l'enfant sont invités à raconter ensemble un livre sans texte. La consigne était la suivante : «Voilà un livre. Nous voudrions que vous le regardiez et que vous le racontiez ensemble. Faites comme vous avez l'habitude de faire. Prenez le temps nécessaire.». Les participants doivent donc co-construire une histoire à partir des images du livre.

Dans la situation de jeu symbolique, à partir d'une maison et d'une ferme playmobil ${ }^{\circledR}$ et d'un certain nombre d'objets et de personnages prédéterminés, les participants doivent jouer ensemble tout en imaginant une histoire. La consigne était la suivante : «Voici une maison avec des personnages, des animaux et d'autres objets. Nous aimerions que vous jouiez ensemble, en imaginant une histoire. Vous avez un quart d'heure. Ne vous inquiétez pas du temps, on vous avertira quelques minutes avant la fin.»

13 Ces deux situations ont des caractéristiques spécifiques et sont susceptibles d'influencer les productions langagières des participants. Ainsi, contrairement au jeu symbolique, la lecture de livre est une tâche plus contraignante car elle suppose l'actualisation des conduites spécifiques de récit. Dans le cas du jeu symbolique, l'absence de contraintes particulières concernant le genre de discours à produire et son caractère foncièrement ludique et routinier pour des enfants, représentent des facteurs susceptibles d'augmenter la motivation des enfants à y participer activement et de favoriser ainsi l'expression de conduites discursives variées.

\subsection{Recueil des données}

14 Les interactions mère-enfant ont été enregistrées en audio et en vidéo. Les dialogues ont été transcrits intégralement en tenant compte des conduites verbales et non verbales des locuteurs. En plus des gestes communicatifs, certaines conduites non verbales ont été considérées comme importantes selon les situations : par exemple, en situation de lecture de livre, la manipulation du livre (tourner les pages) a été prise en compte, et en situation de jeu symbolique, toutes les manipulations des objets et des personnages ont été considérées. 


\subsection{Axes d'analyse}

Afin d'observer à la fois l'influence de la situation sur la participation des locuteurs à la co-construction du dialogue et des profils dialogiques des locuteurs, nous avons mis en place trois grands axes d'analyse.

Tout d'abord, nous évaluons la participation multimodale et verbale des locuteurs (nombre d'interventions, modalités des interventions, nombre de mots). La seule participation des locuteurs ne nous permettant pas de présenter un profil type des conduites des locuteurs, on s'intéresse, par la suite, à la manière dont les locuteurs participent à la dynamique du dialogue à travers notamment l'analyse des types d'interventions verbales (assertion, question, ordre...). Enfin, la dernière analyse tente de caractériser la participation des enfants quant à la fonction des interventions produites par les locuteurs en observant les rôles pris en charge par les locuteurs selon qu'ils racontent, écoutent, manipulent...

\section{Résultats}

17 L'unité d'analyse des dialogues est l'intervention. On considère comme intervention (verbale, non verbale ou mixte) un ou plusieurs énoncés produits par un même locuteur ayant la même valeur fonctionnelle compte tenu de l'activité en cours. Une même prise de parole peut-être composée de plusieurs interventions.

\subsection{Dynamique de l'interaction}

18 Dans un premier temps, afin d'évaluer la participation des locuteurs au dialogue, nous étudions les interventions produites d'un point de vue quantitatif ainsi que d'un point de vue qualitatif à travers l'analyse des modalités sémiotiques. Les interventions verbales feront l'objet d'une analyse plus approfondie en termes de valeur illocutoire (Austin, 1970 ; Searle, 1972).

\subsubsection{Participation multimodale des locuteurs}

19 De manière générale, en situation de lecture de livre, les mères produisent plus d'interventions que leurs enfants quelle que soit la population. En revanche, les enfants TDL en produisent davantage (39,89\%) que leurs pairs d'âge ( $24,88 \%$ ). (Cf. Figure 1$)$.

Figure 1 - Nombre d'interventions par locuteur et par population en situation de lecture de livre

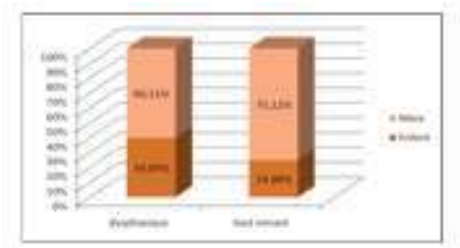

En situation de jeu symbolique, nous constatons que les interactions sont plus symétriques. En effet, même si les mères ont toujours tendance à produire plus d'interventions que leurs enfants, la différence dépasse à peine $50 \%$. De plus, les enfants 
TDL $(47,87 \%)$ et TV $(46,20 \%)$ produisent également presque la même part d'interventions. (Cf. Figure 2)

Figure 2 - Nombre d'interventions par locuteur et par population en situation de jeu symbolique

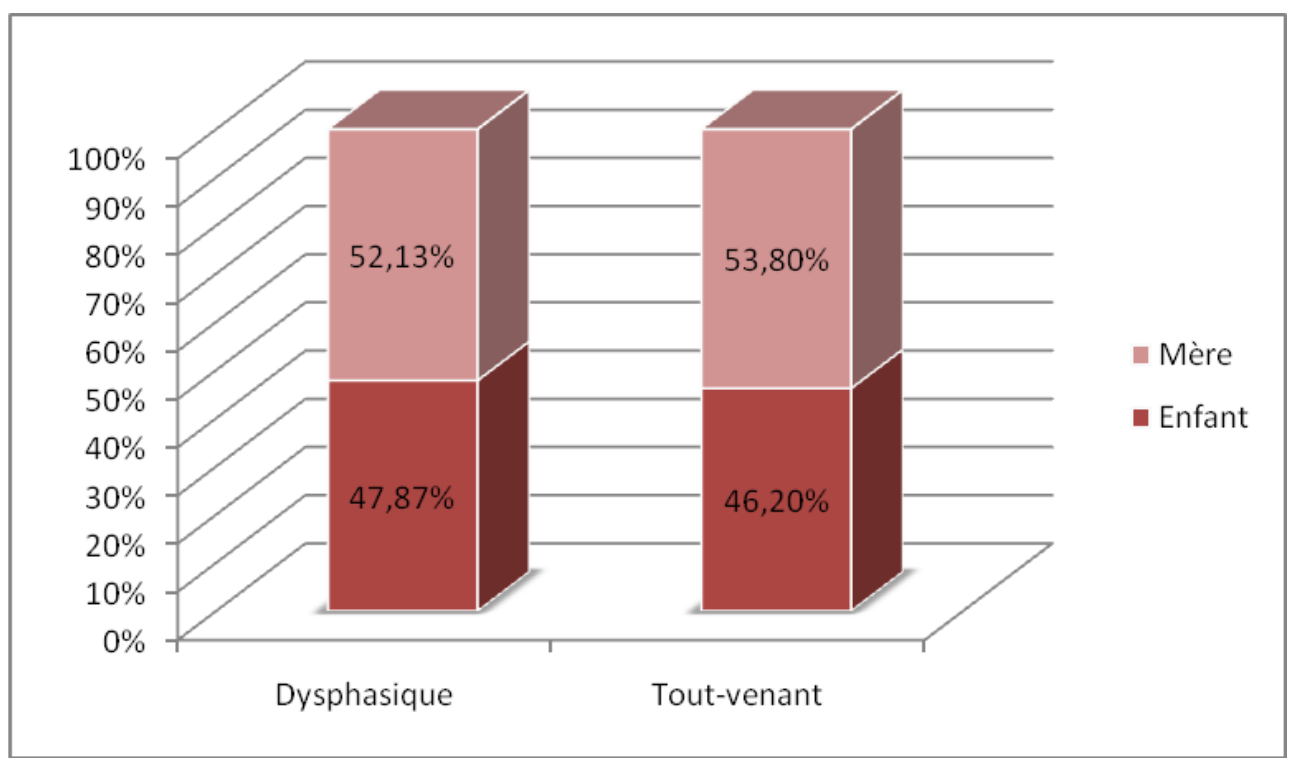

21 De manière générale, l'interaction dans la situation de jeu symbolique semble plus symétrique que celle qui se construit en situation de lecture de livre. Cependant, le nombre d'interventions ne donne qu'un premier aperçu de la participation des locuteurs à la dynamique de l'interaction. Nous nous sommes également interrogés sur la nature de ces interventions, à savoir sur la modalité sémiotique des interventions des locuteurs selon qu'elles sont de nature strictement verbale (Cf. Mère 1 en exemple 1), non verbale (Cf. Enfant 1 en exemple 1) ou mixte (à la fois verbale et non verbale; Cf. Enfant 3 en exemple 1).

MERE 1 - tu vas mettre les messieurs .

ENFANT 1 - ((met un personnage dans un lit))

MERE 2 - donc ça c'est papa : .

ENFANT 2 - ((met un autre personnage dans le même lit))

MERE 3 - là : c'est maman : .

ENFANT 3 - et ça : :à ((montre le personnage à sa mère))

Exemple 1 - Modalités sémiotiques des interventions

Les figures 3 et 4 montrent que les dyades ont des profils relativement semblables en situation de lecture de livre. En effet, tous les locuteurs produisent, de manière croissante, des interventions non verbales, mixtes et verbales. Les interventions verbales représentent une part importante dans le discours des participants, principalement dans les dyades mère-enfant dysphasique. Ces résultats manifestent la nature verbale de cette activité.

On observe cependant quelques différences entre les types de dyades. Par exemple, les enfants TV $(27,70 \%)$ et leurs mères $(23,31 \%)$ produisent plus d'interventions non verbales que les enfants TDL $(17,16 \%)$ et leurs mères $(7,08 \%)$. Toutefois, si on compare le nombre d'interventions non verbales et mixtes des locuteurs, on constate que les enfants TDL et TV ont recours de manière semblable aux gestes alors que les mères des enfants TV utilisent plus fréquemment les gestes que les mères des enfants TDL. 
Figure 3 - Modalités sémiotiques des interventions des enfants dysphasiques et de leurs mères en situation de lecture de livre

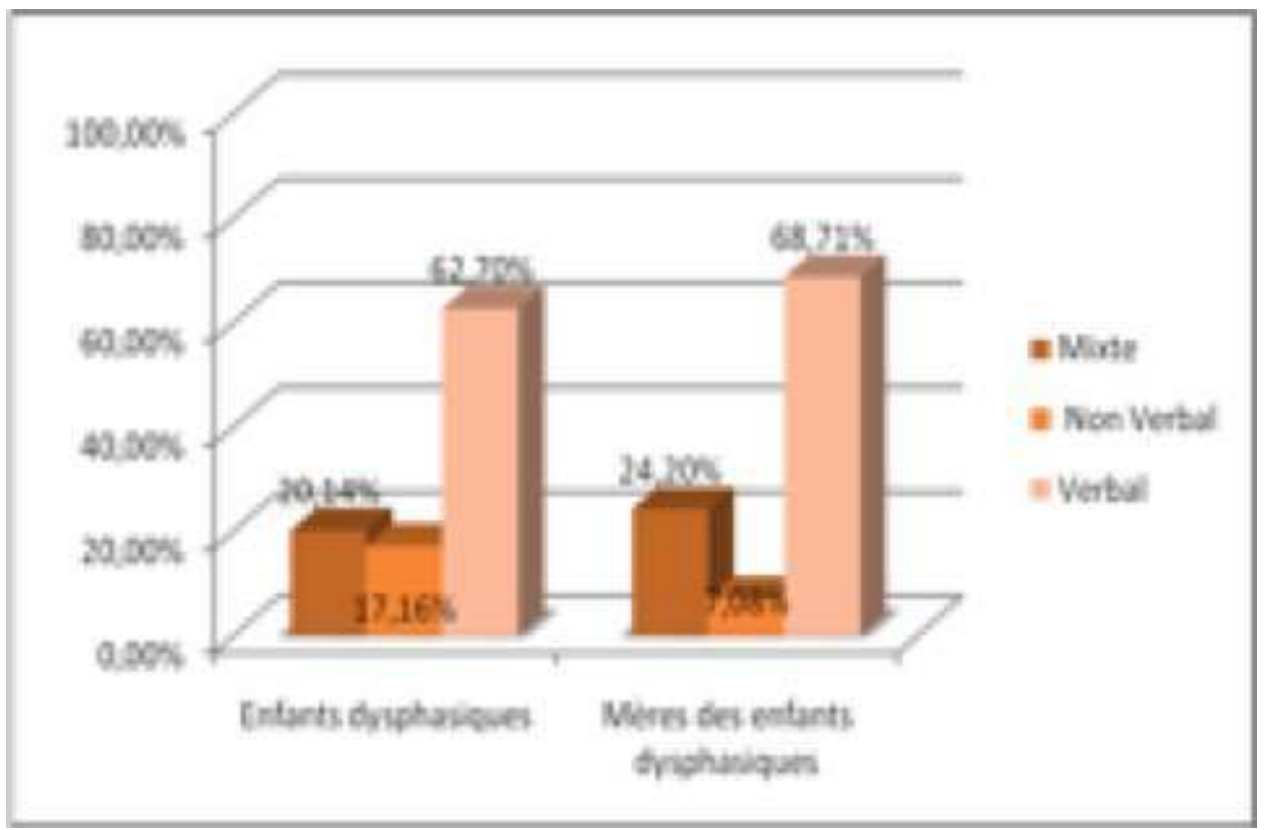

Figure 4 - Modalités sémiotiques des interventions des enfants tout-venant et leurs mères en situation de lecture de livre

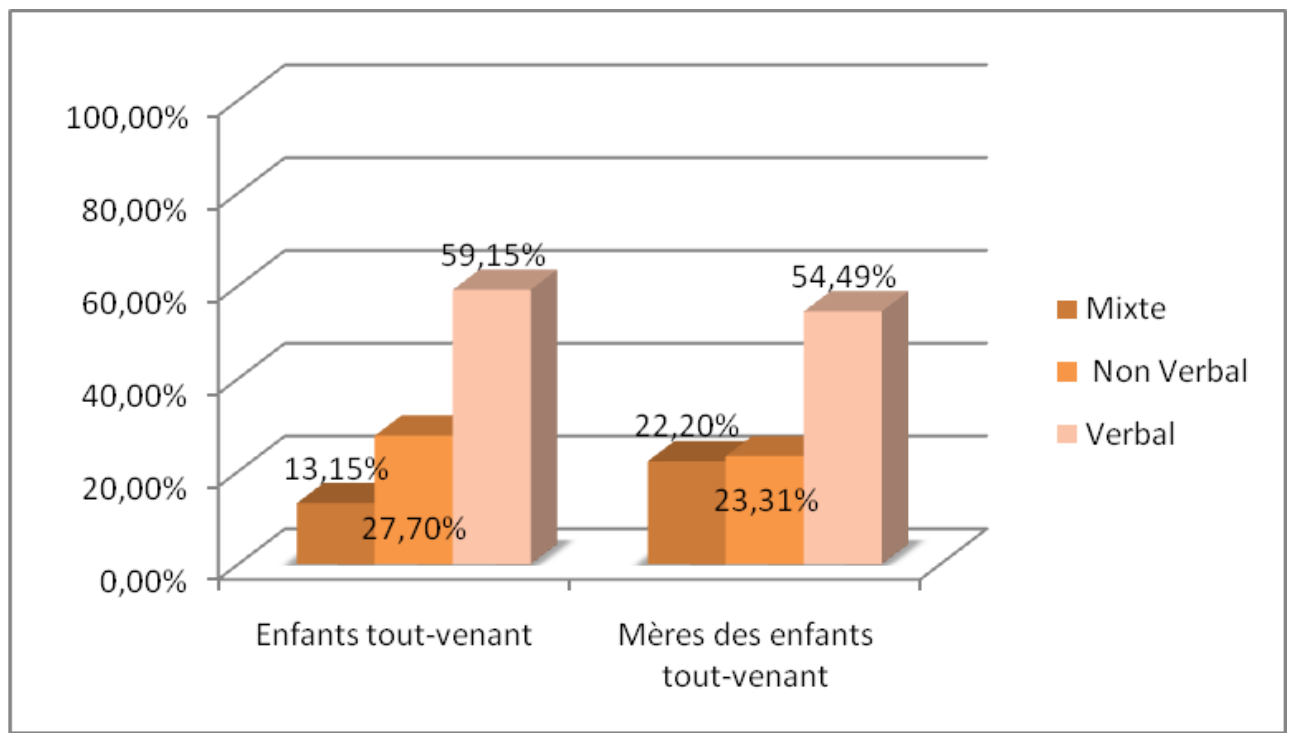

En situation de jeu symbolique (Cf. Figures 5 et 6), des différences sont observées entre les enfants et les mères. En effet, même si les enfants (TDL : 54,48 \%; TV : 45,95\%) et les mères (TDL : 66,27\%; TV : 65,40\%) produisent majoritairement des interventions verbales, les interventions non verbales et mixtes sont plus fréquentes chez les enfants que chez les mères. En effet, l'activité de jeu symbolique est propice à la manipulation des objets et des personnages. Ainsi, le non verbal accompagne très fréquemment des productions verbales. 
Figure 5 - Modalités sémiotiques des interventions des enfants dysphasiques et de leurs mères en situation de jeu symbolique

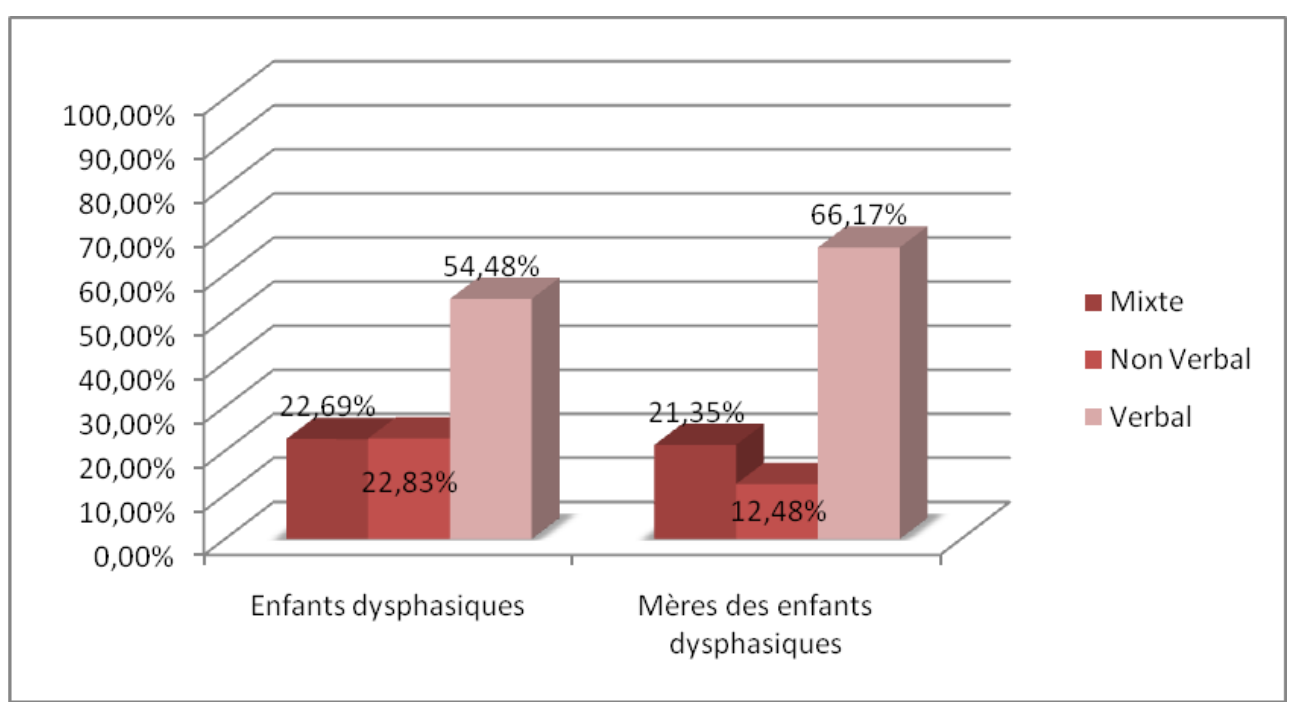

Figure 6 - Modalités sémiotiques des interventions des enfants tout-venant et de leurs mères en situation de jeu symbolique

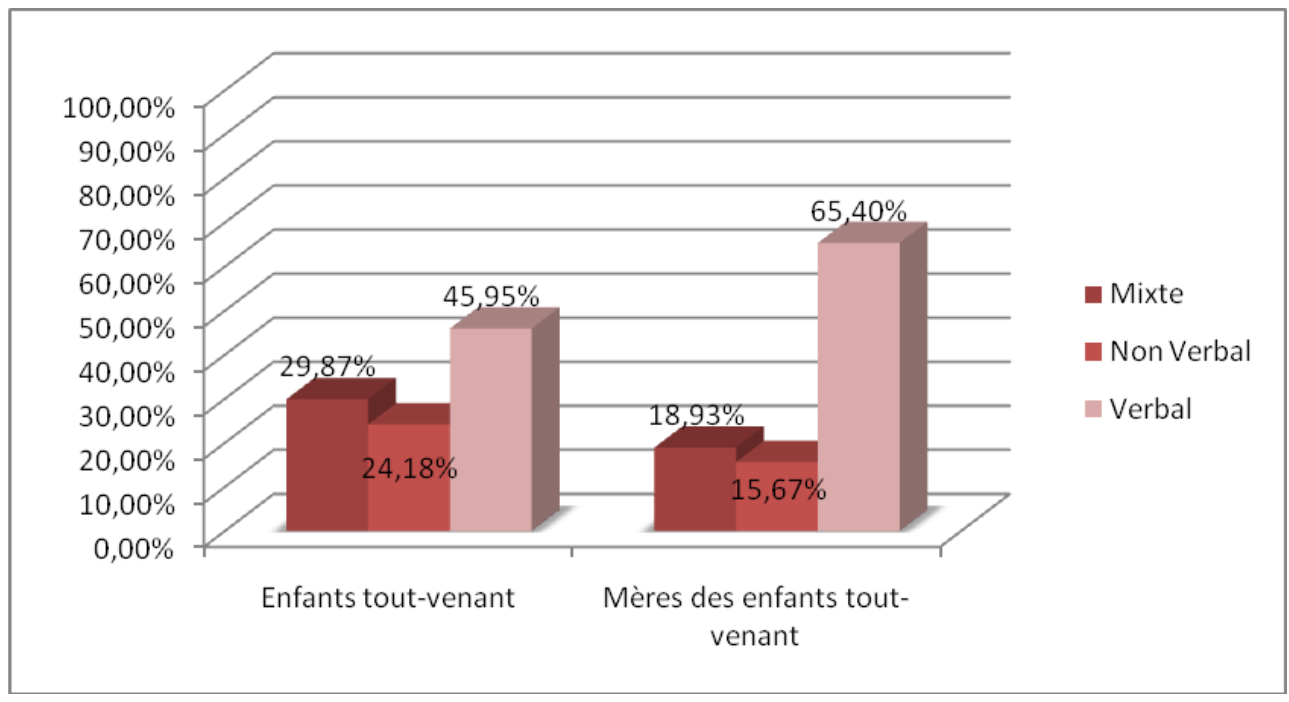

L'analyse des modalités sémiotiques des interventions nous donne des informations à la fois sur la conduite des locuteurs et les activités langagières. En effet, on constate que même si les locuteurs produisent davantage d'interventions verbales, quelle que soit la situation, les taux d'interventions non verbales et mixtes sont plus élevés en situation de jeu symbolique. Cela s'explique par le fait que l'activité de lecture de livre est par nature verbale, alors que l'activité de jeu symbolique est plus propice à la création d'un monde fictif qui passe notamment par la manipulation des objets et des personnages.

\subsubsection{Participation verbale des locuteurs}

Nous avons pris en compte la dimension sémiotique de la communication. De ce point de vue, la participation des enfants dysphasiques diffère peu de celle des enfants tout-venant que ce soit en lecture de livre ou en jeu symbolique. Il nous semble donc important 
d'observer si c'est également le cas au niveau de la participation strictement verbale. Pour évaluer quantitativement la participation verbale des locuteurs, nous avons pris comme mesure le nombre de mots.

La figure 7 montre que les mères des enfants TDL $(82,17 \%)$ et les mères des enfants TV $(91,16 \%)$ participent plus de manière verbale que leurs enfants (TDL : $17,83 \%$; TV : $8,74 \%$ en situation de lecture de livre. On constate donc une totale asymétrie au sein des dyades mère-enfant. Par ailleurs, les enfants dysphasiques participent verbalement plus que les enfants tout-venant.

Figure 7 - Participation verbale des locuteurs dans les interactions mère-enfant en situation de lecture de livre

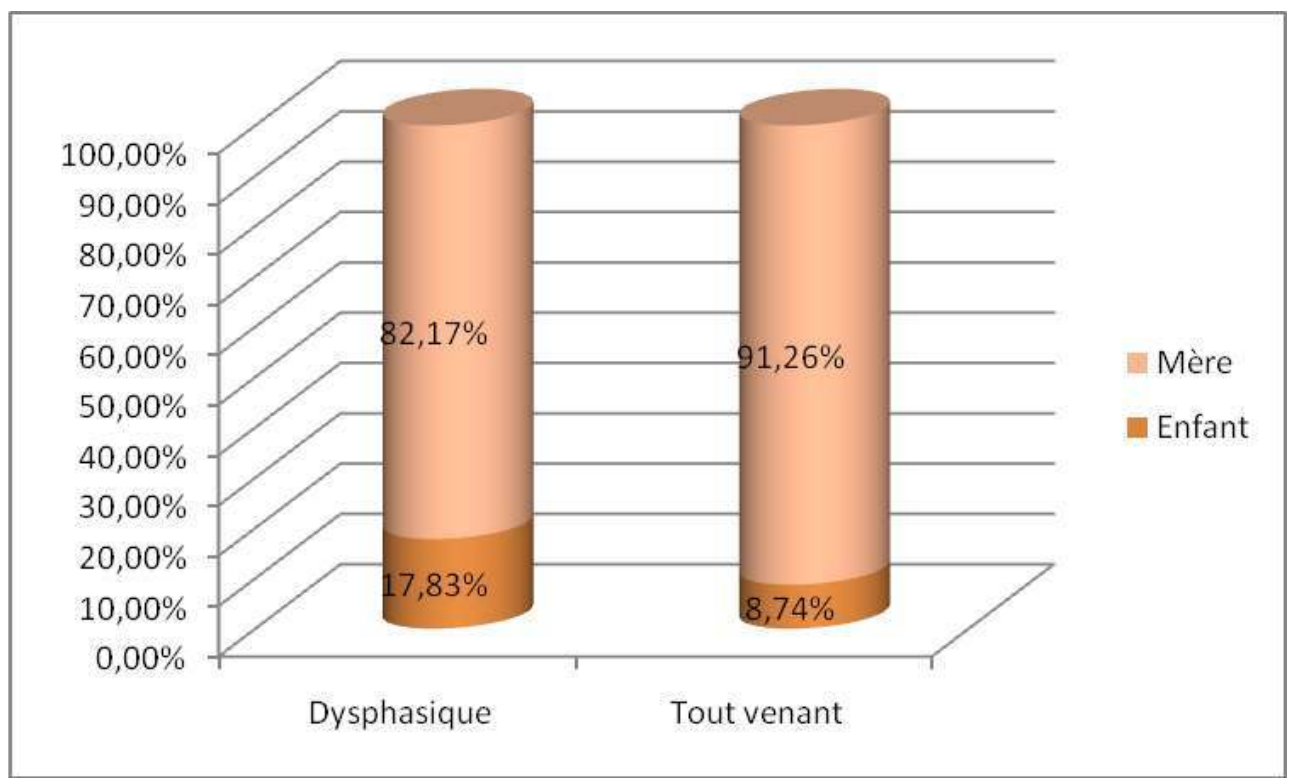

En situation de jeu symbolique, les mères des enfants TDL (70,57 \%) et les mères des enfants TV (66,67\%) participent plus que leurs enfants. La différence entre les enfants TDL $(29,43 \%)$ et TV $(33,33 \%)$ semble moins importante et non significative dans cette situation. 
Figure 8 - Participation verbale des locuteurs dans les interactions mère-enfant dans la situation de jeu symbolique

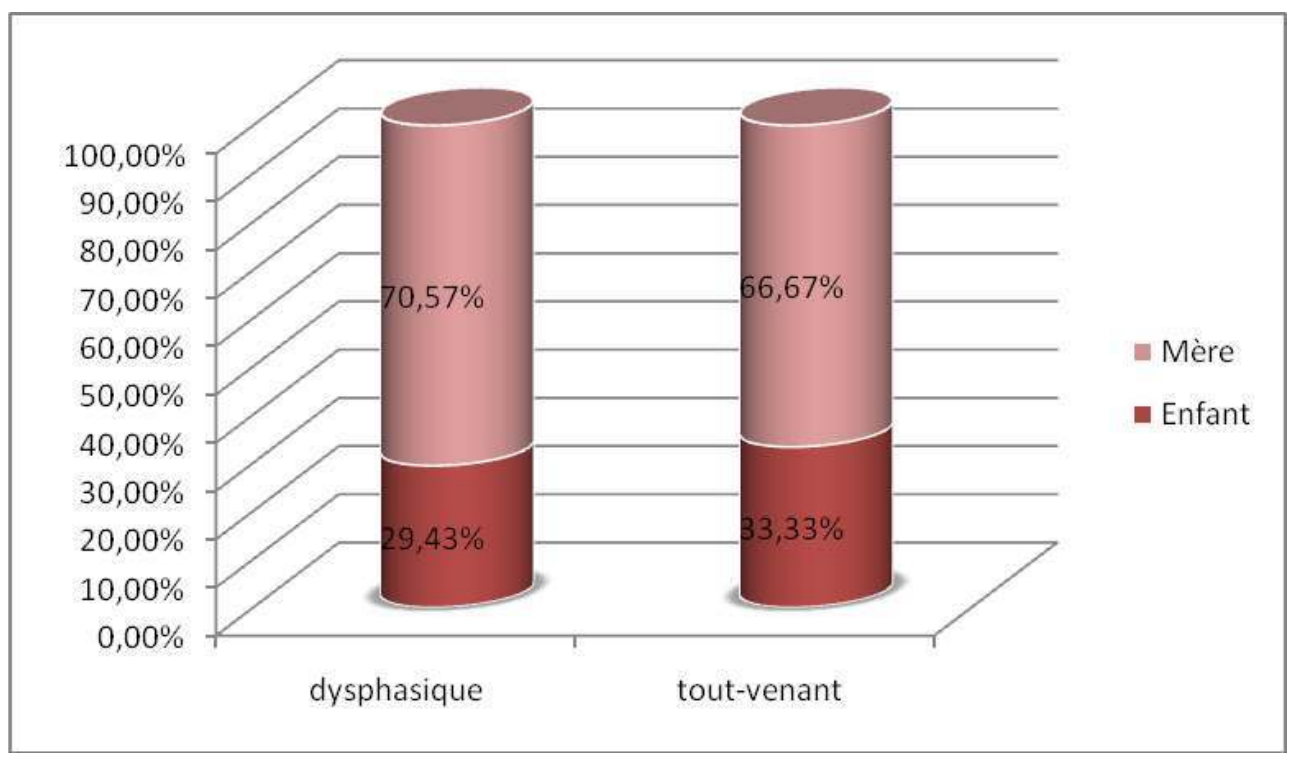

Les résultats concernant la participation verbale des locuteurs ne présentent pas de différence entre les dyades mère-enfant dysphasique et les dyades mère-enfant toutvenant. En effet, alors qu'en situation de lecture de livre, l'interaction est totalement asymétrique entre les mères et les enfants, en situation de jeu symbolique, cette asymétrie est moins prononcée. Les activités ont donc une influence sur la participation strictement verbale des locuteurs.

\subsection{Les types d'interventions verbales}

Comme cela a déjà été décrit dans la littérature (Conti-Ramsden, Hutcheson et Grove, 1995), les enfants dysphasiques participent par des interventions minimales et souvent en position de répondant. Par ailleurs, ces conduites influencent celles de leurs mères dans l'interaction. Elles semblent être plus directives que les mères des enfants tout-venant. Ainsi, nous nous sommes intéressés à la valeur illocutoire des interventions verbales et mixtes des locuteurs.

\subsubsection{Les types d'interventions verbales}

31 Nous avons analysé les interventions verbales et mixtes en six catégories. Ainsi, une intervention verbale peut être :

- une assertion : le locuteur déclare un fait, un évènement, une action...

Exemple 2 - - Intervention verbale de type assertion

Mère 1 - donc on a Frédéric / Chantal // Léo et Charlotte qui habitent cette maison et qui ont $(3 \mathrm{sec})$ un endroit un jardin assez magnifique dans lequel ils ont planté des choses .

- une question: le locuteur produit un énoncé qui a pour finalité d'obtenir de la part de l'interlocuteur une information ou une confirmation, une clarification ... 
Exemple 3 - Intervention verbale de type question

Mère 1 - et alors tu veux faire qui toi ?

- une réponse : il s'agit d'une intervention verbale (de type assertion) qui suit une question, ou un ordre incitant une conduite verbale. (Cf. exemple 3)

Exemple 4 - Intervention verbale de type réponse (ENFANT 1)

Mère 1 - tu veux qu'elle soit dans la chambre des enfants?

Enfant 1 - ouais : ! = ((en mettant la radio dans la chambre des enfants $))$

- un ordre : c'est un acte qui correspond à une demande, une requête ou une prière d'action ou de conduite verbale. Le locuteur produit un énoncé pour demander à son interlocuteur d'accomplir un acte, comme dans l'exemple 5.

Exemple 5 - Interventions verbale de type ordre

Mère 1 - alors vas-y installe-le :i

- un phatique-exclamatif: cette catégorie regroupe les acquiescements et les refus, les phatiques (les régulateurs, back channels...) et les exclamations (interjections, onomatopées) (Cf. Mère 2, Exemple 6).

Exemple 6 - Intervention verbale de type phatique-exclamatif

Mère 1 - c'est sa maman tu crois?

Enfant 1 - oui .

Mère 2 - Très bien. ((tourne la page))

Enfin, une catégorie indécidable a été créée pour les cas de verbal inaudible et les énoncés inachevés.

\subsubsection{Résultats}

En situation de lecture de livre (Cf. Tableau 1 et 2), les mères produisent plus d'assertions (TDL : $63,84 \%$; TV : 64,81\%) que les enfants (TDL : 40,04\%; TV : $21,23 \%$ ).

Par ailleurs, elles adressent plus de questions (TDL : 24,27 \%; TV : 25,31\%) à leurs enfants également. Par conséquent, ces derniers (TDL : 32,60\%; TV : 51,37\%) répondent plus fréquemment à leurs mères. Dans cette situation, les questions posées par les mères permettent de faire participer davantage les enfants à la production du récit.

Les autres types d'interventions verbales (ordre, phatique-exclamatif) sont très peu représentés dans le discours des locuteurs. Cependant, on constate que les enfants TDL ont tendance à produire plus de phatique-exclamatif $(16,85 \%)$ que leurs pairs d'âge $(11,64 \%)$ et les mères des enfants TDL $(3,67 \%)$ produisent plus fréquemment des ordres que les mères des enfants TV $(1,85 \%)$.

Tableau 1- Types d'intervention verbale dans les interactions mère-enfant dysphasique en situation de lecture de livre

\begin{tabular}{|l|l|l|l|l|l|l|l|}
\hline $\begin{array}{l}\text { Types } \\
\text { d'interventions } \\
\text { verbales } \\
\text { Locuteur }\end{array}$ & Assertion & $\begin{array}{l}\text { Phatique - } \\
\text { exclamatif }\end{array}$ & Ordre & Réponse & Question & Indécidable & $\begin{array}{l}\text { Total } \\
\text { général }\end{array}$ \\
\hline $\begin{array}{l}\text { Enfants } \\
\text { dysphasiques }\end{array}$ & $\begin{array}{l}40,04 \% \\
(183)^{1}\end{array}$ & $16,85 \%(77)$ & $\begin{array}{l}1,31 \% \\
(6)\end{array}$ & $\begin{array}{l}32,60 \% \\
(149)\end{array}$ & $\begin{array}{l}3,72 \% \\
(17)\end{array}$ & $5,47 \%(25)$ & $\begin{array}{l}100,00 \% \\
(457)\end{array}$ \\
\hline $\begin{array}{l}\text { Mères des } \\
\text { enfants } \\
\text { dysphasiques }\end{array}$ & $\begin{array}{l}63,84 \% \\
(505)\end{array}$ & $5,69 \%(45)$ & $\begin{array}{l}3,67 \% \\
(29)\end{array}$ & $\begin{array}{l}1,39 \% \\
(11)\end{array}$ & $\begin{array}{l}24,27 \% \\
(192)\end{array}$ & $1,14 \%(9)$ & $\begin{array}{l}100,00 \% \\
(791)\end{array}$ \\
\hline
\end{tabular}




\begin{tabular}{|l|l|l|l|l|l|l|l|}
\hline Total général & $\begin{array}{l}55,13 \% \\
(688)\end{array}$ & $9,78 \%(122)$ & $\begin{array}{l}2,80 \% \\
(35)\end{array}$ & $\begin{array}{l}12,82 \% \\
(160)\end{array}$ & $\begin{array}{l}16,75 \% \\
(209)\end{array}$ & $2,72 \%(34)$ & $\begin{array}{l}100,00 \% \\
(1248)\end{array}$ \\
\hline
\end{tabular}

Tableau 2 - Types d'intervention verbale dans les interactions mère-enfant tout-venant en situation de lecture de livre

\begin{tabular}{|c|c|c|c|c|c|c|c|}
\hline $\begin{array}{l}\text { Types } \\
\text { d'interventions } \\
\text { verbales } \\
\text { Locuteur }\end{array}$ & Assertion & $\begin{array}{l}\text { Phatique } \\
\text { exclamatif }\end{array}$ & Ordre & Réponse & Question & Indécidable & $\begin{array}{l}\text { Total } \\
\text { général }\end{array}$ \\
\hline $\begin{array}{l}\text { Enfants tout- } \\
\text { venant }\end{array}$ & $\begin{array}{l}21,23 \% \\
(31)\end{array}$ & $11,64 \%(17)$ & $\begin{array}{l}1,37 \% \\
(2)\end{array}$ & $\begin{array}{l}51,37 \% \\
(75)\end{array}$ & $\begin{array}{l}9,59 \% \\
(14)\end{array}$ & $4,79 \%(7)$ & $\begin{array}{l}100,00 \% \\
(146)\end{array}$ \\
\hline $\begin{array}{l}\text { Mères des } \\
\text { enfants tout- } \\
\text { venant }\end{array}$ & $\begin{array}{l}64,81 \% \\
(315)\end{array}$ & $5,35 \%(26)$ & $\begin{array}{l}1,85 \% \\
\text { (9) }\end{array}$ & $\begin{array}{l}2,06 \% \\
(10)\end{array}$ & $\begin{array}{l}25,31 \% \\
(123)\end{array}$ & $0,62 \%(3)$ & $\begin{array}{l}100,00 \% \\
(486)\end{array}$ \\
\hline Total général & $\begin{array}{l}54,75 \% \\
(346)\end{array}$ & $6,80 \%(43)$ & $\begin{array}{l}1,74 \% \\
(11)\end{array}$ & $\begin{array}{l}13,45 \% \\
(85)\end{array}$ & $\begin{array}{l}21,68 \% \\
(137)\end{array}$ & $1,58 \%(10)$ & $\begin{array}{l}100,00 \% \\
(632)\end{array}$ \\
\hline
\end{tabular}

En situation de jeu symbolique (Cf. Tableau 3 et 4), les mères (TDL : 43,73 \% ; TV : 40,84 \%) et les enfants TV ( $40,84 \%)$ produisent plus d'assertions que les enfants TDL (31,24\%).

Ce sont les mères qui adressent des questions (TDL : 30,02\%; TV : $28,40 \%$ ) à leurs interlocuteurs et les enfants (TDL : 30,05\%; TV : 30,64\%) qui y répondent.

Les enfants TDL se distinguent des enfants TV seulement par la production plus importante de phatique-exclamatif (TDL : $16,52 \% ;$ TV : 8,61 \%).

Tableau 3 - Types d'intervention verbale dans les interactions mère-enfant dysphasique en situation de jeu symbolique

\begin{tabular}{|c|c|c|c|c|c|c|c|}
\hline $\begin{array}{l}\text { Types } \\
\text { d'intervention } \\
\text { verbale } \\
\text { Locuteur }\end{array}$ & Assertion & $\begin{array}{l}\text { Phatique } \\
\text { exclamatif }\end{array}$ & Ordre & Réponse & Question & Indécidable & $\begin{array}{l}\text { Total } \\
\text { général }\end{array}$ \\
\hline $\begin{array}{l}\text { Enfants } \\
\text { dysphasiques }\end{array}$ & $\begin{array}{l}31,24 \% \\
(314)\end{array}$ & $16,52 \%(166)$ & $\begin{array}{l}6,17 \% \\
(62)\end{array}$ & $\begin{array}{l}30,05 \% \\
(302)\end{array}$ & $8,76 \%(88)$ & $7,26 \%(73)$ & $\begin{array}{l}100 \% \\
(1005)\end{array}$ \\
\hline $\begin{array}{l}\text { Mères des } \\
\text { enfants } \\
\text { dysphasiques }\end{array}$ & $\begin{array}{l}43,73 \% \\
(558)\end{array}$ & $8,23 \%(105)$ & $\begin{array}{l}10,58 \% \\
(135)\end{array}$ & $\begin{array}{l}4,78 \% \\
(61)\end{array}$ & $\begin{array}{l}30,02 \% \\
(383)\end{array}$ & $2,66 \%(34)$ & $\begin{array}{l}100 \% \\
(1276)\end{array}$ \\
\hline Total général & $\begin{array}{l}38,23 \% \\
(872)\end{array}$ & $11,88 \%(271)$ & $\begin{array}{l}8,64 \% \\
(197)\end{array}$ & $\begin{array}{l}15,91 \% \\
(363)\end{array}$ & $\begin{array}{l}20,65 \% \\
(471)\end{array}$ & $4,69 \%(107)$ & $\begin{array}{l}100 \% \\
(2281)\end{array}$ \\
\hline
\end{tabular}


Tableau 4 - Types d'intervention verbale dans les interactions mère-enfant tout-venant en situation de jeu symbolique

\begin{tabular}{|l|l|l|l|l|l|l|l|}
\hline $\begin{array}{l}\text { Types } \\
\text { d'intervention } \\
\text { verbale } \\
\text { Locuteur }\end{array}$ & Assertion & $\begin{array}{l}\text { Phatique } \\
\text { exclamatif }\end{array}$ & Ordre & Réponse & Question & Indécidable & $\begin{array}{l}\text { Total } \\
\text { général }\end{array}$ \\
\hline $\begin{array}{l}\text { Enfants tout- } \\
\text { venant }\end{array}$ & $\begin{array}{l}40,10 \% \\
(233)\end{array}$ & $8,61 \%(50)$ & $\begin{array}{l}6,54 \% \\
(38)\end{array}$ & $\begin{array}{l}30,64 \% \\
(178)\end{array}$ & $9,47 \%(55)$ & $4,65 \%(27)$ & $\begin{array}{l}100 \% \\
(581)\end{array}$ \\
\hline $\begin{array}{l}\text { Mères des } \\
\text { enfants tout- } \\
\text { venant }\end{array}$ & $\begin{array}{l}40,84 \% \\
(312)\end{array}$ & $11,39 \%(87)$ & $\begin{array}{l}10,08 \% \\
(77)\end{array}$ & $\begin{array}{l}6,81 \% \\
(52)\end{array}$ & $\begin{array}{l}28,40 \% \\
(217)\end{array}$ & $2,49 \%(19)$ & $\begin{array}{l}100 \% \\
(764)\end{array}$ \\
\hline Total général & $\begin{array}{l}40,52 \% \\
(545)\end{array}$ & $10,19 \%(137)$ & $\begin{array}{l}8,55 \% \\
(115)\end{array}$ & $\begin{array}{l}17,10 \% \\
(230)\end{array}$ & $\begin{array}{l}20,22 \% \\
(272)\end{array}$ & $3,42 \%(46)$ & $\begin{array}{l}100 \% \\
(1345)\end{array}$ \\
\hline
\end{tabular}

La variable 'situation' a une influence sur la production des interventions verbales des locuteurs. En effet, on constate par exemple que les locuteurs produisent plus d'ordres dans la situation de jeu symbolique que dans la situation de lecture de livre. On peut faire l'hypothèse qu'en situation de lecture de livre, les locuteurs ont tendance à se restreindre à la description d'images ou à la production du récit par la production d'assertions, alors que la situation de jeu symbolique, plus proche d'une conversation spontanée, amène les participants à produire davantage d'interventions verbales diversifiées. Ainsi, il est important de vérifier cette hypothèse en regardant quelles sont les activités prises en charge par les locuteurs dans ces deux situations, notamment à travers l'analyse des rôles des participants.

\subsection{Rôles des participants dans les interactions}

Les activités langagières que nous observons ici ont à la fois des caractéristiques communes et des spécificités. Au sein des deux activités, les participants doivent raconter une histoire. Cependant, dans l'activité de lecture de livre, ils sont dans l'obligation conventionnelle de suivre les images représentées, alors que dans l'activité de jeu symbolique, la contrainte est seulement exercée par les objets et les personnages présents mais les actions et les objets de discours ne sont pas induits. Par ailleurs, au sein de cette dernière, l'objectif est double : jouer et raconter. Ainsi, les participants doivent préparer la « scène » de jeu. Ils peuvent donc endosser différents rôles dans cette activité.

\subsubsection{Rôles des locuteurs : catégories d'analyse}

41 L'analyse du rôle du locuteur dans les deux situations de communication a porté sur cinq grandes catégories selon si les locuteurs racontent l'histoire (contribution verbale et/ou non verbale de contenu effectuée de manière autonome ou sur incitation), écoutent l'autre (avec éventuellement des marques de réception de type $\mathrm{mhm}$ ), reprennent en écho des verbalisations de l'interlocuteur sans apport d'informations nouvelles, gèrent 
l'activité ou s'ils manipulent le livre (tourner les pages du livre, par exemple ) ou le matériel et les objets du jeu symbolique. (Cf. exemple 7 et 8 )

Exemple 7 - Séquence de lecture de livre dans une interaction mère-enfant dysphasique :

Mère $1-[. .$.$] mais qu'est-ce qu'ils font là ? = ((pointe l'image))$

Enfant 1 - ils cueillent des fleurs .

Mère 2 - oui c'est joli mais je pense pas que ce soient des fleurs je pense pas que ce

soit des : : : s- des : fruits j'en sais rien

Enfant 2 - des [p[55:[0]

Mère 3 - oui je pense que c'est des pommes ((tourne la page)) tu vois i \{viens / va\} les aider à ramasser les fruits avec eux ((dessine un cercle au dessus de l'image)) on dirait plutôt des oranges là ((tourne la page))

Exemple 8 - Séquence de jeu symbolique dans une interaction mère-enfant toutvenant :

Enfant 1 - ((place des objets dans la ferme, $10 \mathrm{sec}))$ je t'aime maman !

Mère 1 - hu :m et bah moi aussi chéri . = ((en continuant de placer des objets dans la maison))

[...]

Mère 2 - $((\text { rire }))^{\circ}((\text { cherche des objets et continue de ranger la maison }))^{\circ}$

Enfant $2-^{\circ}((\text { continue de s'occuper de la ferme }))^{\circ}$

Mère 3 - bon alors qu'est-ce que tu fais avec tes animaux ? ils sont tous ensemble?

Enfant 3 - oui maman . / dans une ferme (il) y a beaucoup d'animaux hein : ? tu sais : : ? = ((continue à placer les animaux dans l'enclos))

\subsubsection{Résultats}

Les tableaux 5 et 6 montrent que, quelle que soit la dyade, les participants ont tendance à se centrer sur la narration lors de l'activité de lecture de livre. Cette tendance est plus accentuée dans le comportement des mères (TDL : 79,58 \%; TV : 71,09\%) alors que les profils des enfants tendent à être plus diversifiés : ils racontent (TDL : 54,58\% ;V : 50,93\%), ils écoutent (TDL : 20,73\%; TV : $25 \%$ ) et ils manipulent le livre (TDL : 16,93\%; TV : $17,13 \%)$.

On remarque que les enfants dysphasiques et les enfants tout-venant ont un profil quasiment identique alors que le profil de leurs mères se différencie légèrement. En effet, les mères des enfants TDL se centrent davantage sur l'activité verbale alors que chez les mères des enfants tout-venant la manipulation du livre prend également une certaine place dans l'activité (24,80\%).

On observe également que les mères des enfants TDL reprennent plus souvent $(5,87 \%)$ les interventions verbales précédentes de leurs enfants que les mères des enfants TV $(1,11 \%$ ).

Tableau 5 - Rôles des enfants dysphasiques et de leurs mères en situation de lecture de livre

\begin{tabular}{|l|l|l|l|l|l|l|l|}
\hline $\begin{array}{l}\text { Rôles } \\
\text { Locuteur }\end{array}$ & Ecoute & Gestion & Manipulation & Raconte & Reprend & Indécidable & $\begin{array}{l}\text { Total } \\
\text { général }\end{array}$ \\
\hline $\begin{array}{l}\text { Enfants } \\
\text { dysphasiques }\end{array}$ & $\begin{array}{l}20,73 \% \\
(120)\end{array}$ & $\begin{array}{l}2,59 \% \\
(15)\end{array}$ & $16,93 \%(98)$ & $\begin{array}{l}54,58 \% \\
(316)\end{array}$ & $\begin{array}{l}4,66 \% \\
(27)\end{array}$ & $0,52 \%(3)$ & $\begin{array}{l}100,00 \% \\
(579)\end{array}$ \\
\hline
\end{tabular}




\begin{tabular}{|l|l|l|l|l|l|l|l|}
\hline $\begin{array}{l}\text { Mères } \\
\text { enfants } \\
\text { dysphasiques }\end{array}$ & $\begin{array}{l}2,35 \% \\
(20)\end{array}$ & $\begin{array}{l}2,93 \% \\
(25)\end{array}$ & $9,27 \%(79)$ & $\begin{array}{l}79,58 \% \\
(678)\end{array}$ & $\begin{array}{l}5,87 \% \\
(50)\end{array}$ & $0,00 \%(0)$ & $\begin{array}{l}100,00 \% \\
(858)\end{array}$ \\
\hline Total générale & $\begin{array}{l}9,78 \% \\
(140)\end{array}$ & $\begin{array}{l}2,80 \% \\
(40)\end{array}$ & $12,37 \%(177)$ & $\begin{array}{l}69,46 \% \\
(994)\end{array}$ & $\begin{array}{l}5,38 \% \\
(77)\end{array}$ & $0,21 \%(3)$ & $\begin{array}{l}100,00 \% \\
(1431)\end{array}$ \\
\hline
\end{tabular}

Tableau 6 - Rôles des enfants tout-venant et de leurs mères en situation de lecture de livre

\begin{tabular}{|l|l|l|l|l|l|l|l|}
\hline $\begin{array}{l}\text { Rôles } \\
\text { Locuteur }\end{array}$ & Ecoute & Gestion & Manipulation & Raconte & Reprend & Indécidable & $\begin{array}{l}\text { Total } \\
\text { général }\end{array}$ \\
\hline $\begin{array}{l}\text { Enfants tout- } \\
\text { venant }\end{array}$ & $\begin{array}{l}25,00 \% \\
(54)\end{array}$ & $\begin{array}{l}2,78 \% \\
(6)\end{array}$ & $17,13 \%(37)$ & $\begin{array}{l}50,93 \% \\
(110)\end{array}$ & $2,78 \%(6)$ & $1,39 \%(3)$ & $\begin{array}{l}100,00 \% \\
(216)\end{array}$ \\
\hline $\begin{array}{l}\text { Mères des } \\
\text { enfants tout- } \\
\text { venant }\end{array}$ & $\begin{array}{l}0,79 \% \\
(5)\end{array}$ & $\begin{array}{l}2,21 \% \\
(14)\end{array}$ & $24,80 \%(157)$ & $\begin{array}{l}71,09 \% \\
(450)\end{array}$ & $1,11 \%(7)$ & $0,00 \%(0)$ & $\begin{array}{l}100,00 \% \\
(633)\end{array}$ \\
\hline Total générale & $\begin{array}{l}6,95 \% \\
(59)\end{array}$ & $\begin{array}{l}2,36 \% \\
(20)\end{array}$ & $22,85 \%(194)$ & $\begin{array}{l}65,96 \% \\
(560)\end{array}$ & $\begin{array}{l}1,53 \% \\
(13)\end{array}$ & $0,35 \%(3)$ & $\begin{array}{l}100,00 \% \\
(849)\end{array}$ \\
\hline
\end{tabular}

En situation de jeu symbolique, on constate que les profils des locuteurs tendent à être semblables, quelle que soit la population (Cf. Tableau 7 et 8). Les taux de « manipulation » et de "raconter" sont prédominants. On constate que les dyades mère-enfant toutvenant (enfant : 63,04 \% ; mère : 58,77 \%) manipulent davantage que les dyades mèreenfant dysphasique (enfant : 52,19\% ; mère : 52,26 \%).

Les mères des enfants dysphasiques reprennent davantage les interventions verbales précédemment produites par leurs enfants (4,25\%) que les mères des enfants tout-venant $(2,19 \%)$.

Tableau 7 - Rôles des enfants dysphasiques et de leurs mères en situation de jeu symbolique

\begin{tabular}{|l|l|l|l|l|l|l|l|}
\hline $\begin{array}{l}\text { Rôles } \\
\text { Locuteur }\end{array}$ & Ecoute & Gestion & Manipulation & Raconte & Reprend & Indécidable & $\begin{array}{l}\text { Total } \\
\text { général }\end{array}$ \\
\hline $\begin{array}{l}\text { Enfants } \\
\text { dysphasiques }\end{array}$ & $\begin{array}{l}4,39 \% \\
(58 \%)\end{array}$ & $\begin{array}{l}5,22 \% \\
(69)\end{array}$ & $52,19 \%(690)$ & $\begin{array}{l}26,93 \% \\
(356)\end{array}$ & $\begin{array}{l}4,99 \% \\
(66)\end{array}$ & $6,28 \%(83)$ & $\begin{array}{l}100,00 \% \\
(1322)\end{array}$ \\
\hline $\begin{array}{l}\text { Mères } \\
\text { enfants } \\
\text { dysphasiques }\end{array}$ & $\begin{array}{l}2,54 \% \\
(37)\end{array}$ & $\begin{array}{l}5,56 \% \\
(81)\end{array}$ & $52,26 \%(762)$ & $\begin{array}{l}33,26 \% \\
(485)\end{array}$ & $\begin{array}{l}4,25 \% \\
(62)\end{array}$ & $2,13 \%(31)$ & $\begin{array}{l}100,00 \% \\
(1458)\end{array}$ \\
\hline Total générale & $\begin{array}{l}3,42 \% \\
(95)\end{array}$ & $\begin{array}{l}5,40 \% \\
(150)\end{array}$ & $52,23 \%(1452)$ & $\begin{array}{l}30,25 \% \\
(841)\end{array}$ & $\begin{array}{l}4,60 \% \\
(128)\end{array}$ & $4,10 \%(114)$ & $\begin{array}{l}100,00 \% \\
(2780)\end{array}$ \\
\hline
\end{tabular}


Tableau 8 - Rôles des enfants tout-venant et de leurs mères en situation de jeu symbolique

\begin{tabular}{|l|l|l|l|l|l|l|l|}
\hline $\begin{array}{l}\text { Rôles } \\
\text { Locuteur }\end{array}$ & Ecoute & Gestion & Manipulation & Raconte & Reprend & Indécidable & $\begin{array}{l}\text { Total } \\
\text { général }\end{array}$ \\
\hline $\begin{array}{l}\text { Enfants } \\
\text { dysphasiques }\end{array}$ & $\begin{array}{l}2,56 \% \\
(20)\end{array}$ & $\begin{array}{l}5,50 \% \\
(43)\end{array}$ & $63,04 \%(493)$ & $\begin{array}{l}23,02 \% \\
(180)\end{array}$ & $\begin{array}{l}2,30 \% \\
(18)\end{array}$ & $3,58 \%(28)$ & $\begin{array}{l}100,00 \% \\
(782)\end{array}$ \\
\hline $\begin{array}{l}\text { Mères } \\
\text { enfants } \\
\text { dysphasiques }\end{array}$ & $\begin{array}{l}2,96 \% \\
(27)\end{array}$ & $\begin{array}{l}5,59 \% \\
(51)\end{array}$ & $58,77 \%(536)$ & $\begin{array}{l}28,29 \% \\
(258)\end{array}$ & $\begin{array}{l}2,19 \% \\
(20)\end{array}$ & $2,19 \%(20)$ & $\begin{array}{l}100,00 \% \\
(912)\end{array}$ \\
\hline Total générale & $\begin{array}{l}2,77 \% \\
(47)\end{array}$ & $\begin{array}{l}5,55 \% \\
(94)\end{array}$ & $60,74 \%(1029)$ & $\begin{array}{l}25,86 \% \\
(438)\end{array}$ & $\begin{array}{l}2,24 \% \\
(38)\end{array}$ & $2,83 \%(48)$ & $\begin{array}{l}100,00 \% \\
(1694)\end{array}$ \\
\hline
\end{tabular}

Que ce soit en situation de lecture de livre ou de jeu symbolique, les mères des enfants dysphasiques reprennent davantage les interventions verbales de leurs enfants que les mères des enfants tout-venant. Ces reprises en écho sont principalement produites soit comme des reformulations langagières soit dans le but de s'assurer qu'il y a bien intercompréhension soit de produire un feed-back positif. On peut donc penser que cette conduite des mères des enfants TDL est liée aux difficultés linguistiques des enfants TDL.

\section{Conclusion}

L'objectif de cet article était double. Premièrement, nous voulions observer si la participation des enfants dysphasiques à la co-construction du dialogue est semblable ou non à celle des enfants tout-venant de même âge, et voir si les troubles linguistiques des enfants dysphasiques affectent leurs capacités pragmatiques. Deuxièmement, nous souhaitions montrer que la situation de communication influence la manière dont les locuteurs participent à la co-construction du dialogue.

De manière générale, on a remarqué que les enfants dysphasiques et tout-venant ont des profils qui tendent à être similaires, principalement en situation de jeu symbolique. Quelques différences sont perceptibles entre les enfants TDL et TV en situation de lecture de livre (notamment au niveau de la participation verbale, des types d'interventions verbales). Cependant, malgré leurs difficultés linguistiques, on constate que les enfants TDL présentent une certaine sensibilité aux variations de conditions de production.

50 Par ailleurs, les difficultés linguistiques des enfants dysphasiques peuvent influencer le discours de leurs mères (par exemple, un taux plus élevé d'ordres, de reprises...). Ces ajustements maternels sont plus visibles au sein de l'activité de récit, ce qui rejoint les conclusions d'une analyse plus fine de l'étayage verbal et de l'étayage lié à la tâche à accomplir dans ces situations (Ingold et al., 2008).

51 A travers les résultats obtenus et les différences observées entre les deux situations, on peut se demander si un autre facteur ne joue pas un rôle important dans les conduites dialogiques des locuteurs tel que la représentation que les interlocuteurs ont de l'activité en cours. Par exemple, la forte participation verbale des mères en situation de lecture de livre peut s'expliquer par la représentation que ces mêmes locuteurs ont de cette activité. 
En effet, on peut apparenter l'activité de lecture de livre à une routine où la mère raconte et l'enfant écoute. Ce qui a été vérifié par l'analyse des rôles des participants dans l'interaction.

Pour conclure, il nous semble que la situation de jeu symbolique est une activité qui permet aux enfants TDL d'y accéder par différents genres de discours contrairement à la situation de lecture de livre qui contraint les locuteurs à identifier des actions, des personnages, à constituer une histoire en relation avec les images représentées. Ces contraintes sont liées à la structuration même du récit. Ceci suggère donc qu'il est important de maintenir des situations de communication variées, notamment lors des séances de rééducation, afin de pouvoir considérer tous les aspects du langage et de s'inscrire dans divers genres de discours.

\section{BIBLIOGRAPHIE}

Austin, J.L. (1970), Quand dire c'est faire, Paris, Edition du Seuil.

Bishop, D. V. M. (2000), « Pragmatic language impairment: A correlate of SLI, a distinct subgroup, or part of the autistic continuum? », in D.V.M. Bishop \& L.B. Leonard (Eds.), Speech and language impairments in children: causes, characteristics, intervention and outcome, Hove, Psychology Press, p. 99-113.

Bishop, D. V. M. et Adams, C. (1989), « Conversational characteristics of children with semanticpragmatic disorder. II: What features lead to a judgement of inappropriacy? » The British Journal of Disorders of Communication 24: 241-263.

Bishop, D.V.M., Chan, J., Adams, C, Hartley, J. \& Weir, F. (2000). « Conversational responsiveness in specific language impairment: Evidence of disproportionate pragmatic difficulties in a subset of children ». Development and Psychopathology, 12, p. 177-190.

Brinton, B. et Fujiki, M. (1982), « A comparison of request-response sequences in the discourse of normal and language-disordered children ", Journal of Speech and Hearing Disorders 47, p. 57-62.

Brinton, B., Fujiki, M. et Sonnenberg, E. (1988), « Responses to requests for clarification by linguistically normal and language impaired children in conversation », Journal of Speech and Hearing Disorders 53: 383-391.

Conti-Ramsden, G. (1994), « Language interaction with atypical language learners », in C. Gallaway \& B. Richards (Eds.), Input and Interaction in Language Acquisitio, Cambridge, CUP, p. 183-196.

Chevrie-Muller, C., Plaza, M., Fournier, S., et al., (2001), N-EEL : nouvelles épreuves pour l'examen du langage, Paris, Les Editions du Centre de Psychologie Appliquée.

Conti-Ramsden, G. et Friel-Patti, S. (1984), « Mother-child dialogues: a comparison of normal and language impaired children », Journal of Communication Disorders 84 (17), p. 19-35.

Conti-Ramsden, G. \& Gunn, M., (1986), « The development of conversational ability: A case study », British Journal of Disorders of Communication, 21, p. 339-351. 
Conti-Ramsden, G., Hutcheson, G.D. \& Grove, J. (1995), « Contingency and breakdown: Children with SLI and their conversations with mothers and fathers ». Journal of Speech and Hearing Research , 38, 1290-1302.

de Weck, G., (1996), « Troubles du développement du langage : de la structure au discours », in G. de Weck (Ed.), Troubles du développement du langage. Perspectives pragmatiques et discursives, Lausanne-Paris : Delachaux et Niestlé, p. 189-246.

de Weck, G. et Rosat, M. C., (2003), Troubles dysphasiques. Comment raconter, relater, faire agir à l'âge préscolaire, Paris, Masson.

Dolz, J., Pasquier, A., \& Bronckart, J.-P. (1993), « L'acquisition des discours : émergence d'une compétence ou apprentissage de capacités langagières diverses ? Études de linguistique appliquée, 92, p. 23-37.

Fujiki, M., Brinton, B. et Sonnenberg, E., (1990), « Repair of overlapping speech in the conversations of specifically language-impaired and normally developing children », Applied Psycholinguistics 11, p. 201-215

Gérard, C.L. (1991), L'enfant dysphasique, Paris, Editions Universitaires.

Hupet, M. (1996), « Troubles de la compétence pragmatique : troubles spécifiques ou dérivés? », in G. de Weck (Ed.), Troubles du développement du langage. Perspectives pragmatiques et discursives, Lausanne-Paris, Delachaux et Niestlé p. 89-112.

Ingold, J., Gendre, S, Rezonnico, S., Corlateanu, C. et da Silva, C. (2008), « Diversité des étayages des mères des enfants tout-venant et dysphasiques dans deux situations d'interaction ", Travaux Neuchâtelois de Linguistique, 49, p. 69-82.

Leonard, L. B. (1986), « Conversational replies of children with specific language impairment », Journal of Speech and Hearing Disorders 29, p. 114-119.

Radford , J.\& Tarplee, C., (2000), « The management of conversational topic by a ten years old child with pragmatic difficulties », Clinical Linguistics and Phonetics, 14 (5), p. 387-403.

Rapin, I. \& Allen, D.A. (1983), « Developmental language disorders », in U. Kirk (Ed.), Neuropsychology of language, reading and spelling, New York, Academic Press, p. 155-184.

Searle, J. (1972). Les actes de langage, Paris, Hermann.

\section{ANNEXES}

\section{Conventions de transcription :}

. assertion

? interrogation

! exclamation

j ordre

$\rightarrow$ énoncé en suspens

+ ébauche

// pause 
= simultanéité entre verbal et non verbal

(()) non verbal

[]: transcription phonétique

\section{NOTES}

1. Les valeurs entre parenthèse dans les tableaux de cet article correspondent aux valeurs absolues.

\section{RÉSUMÉS}

Cet article présente les premiers résultats d'une recherche plus vaste portant sur les interactions mère-enfant dysphasiques dans différentes situations de communication Afin d'observer si la situation peut influencer la manière dont les enfants dysphasiques participent à la coconstruction $\mathrm{du}$ dialogue avec leurs mères et si cette participation diffère de celle des enfants sans troubles du développement du langage, nous avons étudié les interactions mère-enfant dans deux situations de communication (une lecture de livre et un jeu symbolique). Les résultats semblent indiquer que la situation influe sur la participation multimodale et verbale des locuteurs, sur le type d'intervention verbale ainsi que sur les rôles pris en charge par les locuteurs.

This paper presents the first results of a larger research on the dialogical behaviours of motherchild dyads in different situations of communication. 4 dyads with specific language impairment children (SLI) aged from 5 to 7 were compared to 4 dyads with normally developing children (ND) matched on age. The aim of this article is to investigate the influence of the situation on the way SLI children co-construct the dialogue with their mothers and if this participation differs from that of normally developing children. We have considered for this study two familiar situations of communication: a symbolic game and a picture book reading activity. Results show that the situation seems to have an influence on the multimodal participation, on the illocutionary types of verbal moves, and on the roles that the participants play in the interaction.

\section{AUTEURS}

\section{CHRISTINE DA SILVA}

Université Paris 3 - Sorbonne Nouvelle / Université de Neuchâtel, Suisse

\section{STEFANO REZZONICO}

Université Paris 3 - Sorbonne Nouvelle / Université de Neuchâtel, Suisse

CRISTINA CORLATEANU

Université Paris 3 - Sorbonne Nouvelle / Université de Neuchâtel, Suisse 


\section{SÉVERINE GENDRE}

Université Paris 3 - Sorbonne Nouvelle / Université de Neuchâtel, Suisse

JULIANE INGOLD

Université Paris 3 - Sorbonne Nouvelle / Université de Neuchâtel, Suisse 